\title{
Optimal Government Spending Ratio on the Research Sector
}

\author{
Zexiong Wu \\ Jinan University, Guangzhou, China \\ Email: hohohowzx@163.com
}

How to cite this paper: Wu, Z.X. (2018) Optimal Government Spending Ratio on the Research Sector. Open Journal of Business and Management, 6, 558-567. https://doi.org/10.4236/ojbm.2018.63042

Received: April 17, 2018

Accepted: May 29, 2018

Published: June 1, 2018

Copyright (C) 2018 by author and Scientific Research Publishing Inc. This work is licensed under the Creative Commons Attribution International License (CC BY 4.0).

http://creativecommons.org/licenses/by/4.0/

\begin{abstract}
This paper investigates the optimal government spending ratio on the research sector by introducing government effects into Romer's endogenous model. The government spending has positive effects on both the final-output sector and the research sector. Under this circumstance, we first show that there exists a minimum government spending proportion to maintain endogenous growth and there are two optimal spending ratios on the research sector given a constant government proportion. Then we analyze how the government chooses between these two ratios. When the government is interested in the balanced growth rate, it will always choose the high spending ratio on the research sector. But since there is a dynamic transition process in our model, short-run welfare must be taken into the government's account. If the stock of endowed knowledge is much more than that of endowed physical capital, then the government will choose the low spending ratio on the research sector.
\end{abstract}

\section{Keywords}

Government Spending, Endogenous Growth, Economic Development

\section{Introduction}

Economic growth is always one of the most attractive and fascinating topics for all economists. Romer (1990) [1] presents how technological change contributes to it. In his model, the motivation of designing a new instruction for working with raw materials is market incentives. Technology is a non-rival yet excludable input, in that a design can be replicated costless compared to the cost of creating it and thus the accumulation of knowledge is beneficial for all innovations. But the design in the first place is compensated out of quasi rents from a single monopolist who invests in technological change and hence gets the market power 
after a new design comes out. In the research sector, only the stock of knowledge and human capital employed account for a new design.

But Barro (1990) [2] incorporates a public sector into a simple constant-returns model of economic growth and shows how familiar externalities of government spending drive the economy to grow. So this paper investigates the effects of government spending on Romer's economy and presents that government spending helps not only in the final-output sector but also in the research sector. Hence there are interesting choices about the spending ratios on both sectors.

Section 2 below presents the whole model of the paper. The first part of it introduces government spending into Romer's model, and gets the optimal dynamic path. The second part then analyzes the balanced growth path, and shows how government chooses spending ratio on the research sector if the equilibrium growth rate is the interest of it. The last part takes short-run analysis into account because there is a dynamic transition process in the model, and derives the relation between government spending ratio on the research sector and the ratio in specific form of endowed stock of knowledge and endowed physical capital.

\section{The Model}

\subsection{Description of the Model}

Romer (1990) [1] described four basic inputs are used in the final-goods sector to produce the final outputs: physical capital, human capital, labor and an index of the level of the technology. Unlike other models, Romer disaggregates physical capital into an infinite number of distinct types of producer durables, and thus assumes that it takes $\eta$ units of $K$ to create one unit of any type of durables. So the final outputs $Y$ is expressed as a function of human capital devoted to final output $H_{Y}$, labor $L$, and the producer durables $x$. In the research sector, only the human capital devoted to research $H_{A}$ and the existing stock of knowledge $A$ are used to produce new knowledge. A new knowledge is used to design a new producer durable.

\subsubsection{Setup of the Model}

In our model, the modification of Romer's model is based on two important premises. First, we assume that government spending can be used into two sectors: the final-goods sector and the research sector, and is a constant proportion of the output. Let $G_{2}$ and $G_{1}$ denote the spending on these two sectors respectively, then

$$
G_{1}+G_{2}=G=g * Y
$$

where $g$ is the constant expenditure proportion.

Second, we do not distinguish human capital and physical labor, and the supply of the labor is constant, denoted as $H$. So our production function of the final-goods is supposed to be

$$
Y=\left(H-H_{A}\right)^{\alpha} G_{2}^{\beta} \int_{0}^{\infty} x(i)^{1-\alpha-\beta} \mathrm{d} i=\eta^{\alpha+\beta-1} A^{\alpha+\beta}\left(H-H_{A}\right)^{\alpha} G_{2}^{\beta} K^{1-\alpha-\beta}
$$

And the aggregate stock of designs evolves according to 


$$
\dot{A}=B\left(\frac{G_{1}}{Y}\right) H_{A} A
$$

where $B$ is constant over time.

Then we consider a standard Ramsey consumer with constant discount rate $\rho$ and $\log$ utility

$$
U=\int_{0}^{\infty} \log C * \mathrm{e}^{-\rho t} \mathrm{~d} t
$$

\subsubsection{Solution of the Model}

Suppose the government chooses the optimal proportion, $u$, of the spending on the research sector, then dictates each household's choice for consumption and labor allocation on these two sector over time. So the final outputs

$$
Y=\eta^{\frac{\alpha+\beta-1}{1-\beta}} A^{\frac{\alpha+\beta}{1-\beta}}\left(H-H_{A}\right)^{\frac{\alpha}{1-\beta}}[(1-u) g]^{\frac{\beta}{1-\beta}} K^{\frac{1-\alpha-\beta}{1-\beta}}
$$

Then we get optimization problem for the central planner

$$
\max _{u, H_{A}, C, K, A} U
$$

Subject to

$$
\begin{aligned}
\dot{K} & =(1-g) Y-C \\
\dot{A} & =B(u g) H_{A} A
\end{aligned}
$$

To solve this problem, we use the Hamiltonian

$$
\mathcal{H}=\log C+\lambda[(1-g) Y-C]+\mu B u g H_{A} A
$$

where $\lambda, \mu$ are the co-state variables for $K$ and $A$ respectively. Then the optimal solution is given by

$$
\begin{gathered}
\frac{1-u}{u}=\frac{\beta}{\alpha} \frac{H-H_{A}}{H_{A}} \\
\frac{\lambda}{\mu}=\frac{1-\beta}{\alpha} \frac{H-H_{A}}{1-g} \frac{B u g A}{Y} \\
\frac{\dot{C}}{C}=-\frac{\dot{\lambda}}{\lambda}=-\left[\rho-(1-g) \frac{1-\alpha-\beta}{1-\beta} \frac{Y}{K}\right] \\
\frac{\dot{\mu}}{\mu}=\rho-\left(\frac{\alpha+\beta}{\alpha} H-\frac{\beta}{\alpha} H_{A}\right) B u g \\
\frac{\dot{K}}{K}=(1-g) \frac{Y}{K}-\frac{C}{K} \\
\frac{\dot{A}}{A}=B u g H_{A}
\end{gathered}
$$

Equations (1) to (6) show the optimal dynamic process of the model.

\subsection{Analysis of the Balanced Growth Path}

\subsubsection{Balanced Growth Equilibrium}

It follows immediately that on the balanced growth path, $u$ and $H_{A}$ are constant, 
and can be solved by

$$
\begin{gathered}
\frac{\alpha}{\alpha+\beta} \rho=B u g\left(H-H_{A}\right) \\
\frac{\alpha}{\alpha+\beta} \rho=B(1-u) g H_{A}
\end{gathered}
$$

Then we have

$$
\frac{\dot{C}}{C}=\frac{\dot{K}}{K}=\frac{\dot{Y}}{Y}=\frac{\alpha+\beta}{\alpha} \frac{\dot{A}}{A}
$$

Let $\gamma$ denote the growth rate of $C, K$ and $Y$, and $\gamma_{a}$ the growth rate of $A$, then

$$
\gamma=\frac{\alpha+\beta}{\alpha} \gamma_{a}=\frac{\alpha+\beta}{\alpha} B u g H-\rho
$$

On the balanced growth path, the ratio between outputs and physical capital, and that between consumption and physical capital are also constant,

$$
\begin{gathered}
\left(\frac{Y}{K}\right)^{*}=\frac{1-\beta}{1-\alpha-\beta} \frac{\alpha+\beta}{\alpha} \frac{B u g H}{1-g} \\
\left(\frac{C}{K}\right)^{*}=\rho+\frac{1-\beta}{1-\alpha-\beta} B u g H
\end{gathered}
$$

\subsubsection{Analysis of the Optimal Spending Ratio on the Research Sector} From differentials of Equations (7) and (8), we have

$$
\begin{gathered}
\frac{\mathrm{d} u}{\mathrm{~d} g}=\frac{B^{2}(1-u) u g H}{\Delta} \\
\frac{\mathrm{d} H_{A}}{\mathrm{~d} g}=\frac{B^{2} g\left(H-H_{A}\right) H_{A}}{\Delta} \\
\frac{\mathrm{d} \gamma_{a}}{\mathrm{~d} g}=\frac{B^{3} u g^{2} H H_{A}}{\Delta}
\end{gathered}
$$

where

$$
\Delta=\frac{B g \rho}{\alpha+\beta}\left(\beta \frac{u}{1-u}-\alpha \frac{1-u}{u}\right)
$$

It follows that on the balanced growth path, $u$, and $H_{A}$ changed in the same direction. So more government spending on the research sector will not squeeze out the labor supply on the research, but incent more labor to research. The signs of $\frac{\mathrm{d} u}{\mathrm{~d} g}, \frac{\mathrm{d} H_{A}}{\mathrm{~d} g}$ and $\frac{\mathrm{d} \gamma_{a}}{\mathrm{~d} g}$ depend on the sign of $\Delta$. From Equations (7) and (8), the planner chooses optimal ratio of the spending $u$ according to

$$
\frac{(1-u) u}{\alpha(1-u)+\beta u}=\frac{\rho}{B H(\alpha+\beta) g}
$$

Since the left part of the equation has a maximum, to make sure there exists an optimal $u$, the government spending ratio $g$ must satisfy 


$$
g \geq g_{\min }=\frac{\rho}{B H(\alpha+\beta)(\sqrt{\alpha}+\sqrt{\beta})^{2}}
$$

If the consumer becomes more patient about future, and the labor supply increases, the minimum government spending will be lower. First, more patient consumer will save more. Second, labor supply in our model has the same effect as government spending does, thus not only help product but also help design. So the country with more patience and more labor supply will depend less on the government spending to maintain endogenous growth.

There are two solutions that satisfies (9) if $g$ is strictly larger than $g_{\min }$, denoted by $\underline{u}, \bar{u}$, and $\underline{u}<\bar{u}$. This can easily be seen from Figure 1 .

Since

$$
\underline{u}<\frac{\sqrt{\alpha}}{\sqrt{\alpha}+\sqrt{\beta}}<\bar{u}
$$

More government spending will increase the spending proportion and labor supply on the research sector, thus brings a higher growth rate. If $u=\bar{u}$, then

$$
\Delta>0, \frac{\mathrm{d} u}{\mathrm{~d} g}>0, \frac{\mathrm{d} H_{A}}{\mathrm{~d} g}>0, \frac{\mathrm{d} \gamma_{a}}{\mathrm{~d} g}>0, \text { thus } \frac{\mathrm{d} \gamma}{\mathrm{d} g}>0
$$

More government spending will decrease the spending proportion and labor supply on the research sector, thus bring a lower growth rate.

If the government chooses the higher spending ratio on the research sector, there is a complement between the overall government spending proportion and the spending ratio on the research sector. So the overall government spending ratio will drive the economy to grow fast, and thus has a trend of increase. On the other hand, when the government chooses the lower one, there is a substitution between the overall government spending proportion and the spending ratio on the research sector. This is because the government spending has both direct effect and indirect effect on the final-output sector. From the perspective of direct effect, more government spending will cause the final output increasing since it is one of four kinds of inputs in the final-output sector. From the perspective of indirect effect, government could spend more on the research sector and thus causes $A$ increasing, larger $A$ in turn helps produce more final output. Since more accumulated knowledge causes innovation more efficient, more government spending on the research sector will make the indirect effect exceed the direct one. But if the government spends less on the research sector, the accumulated knowledge is not sufficient enough to design new knowledge efficiently, the direct effect dominates.

For a given government spending ratio $g$, since

$$
\gamma_{a}=\frac{\beta}{\alpha+\beta} \rho \frac{u}{1-u}
$$

if the government's goal is to bring a fast-growing economy in equilibrium, it will always choose $\bar{u}$. And from 


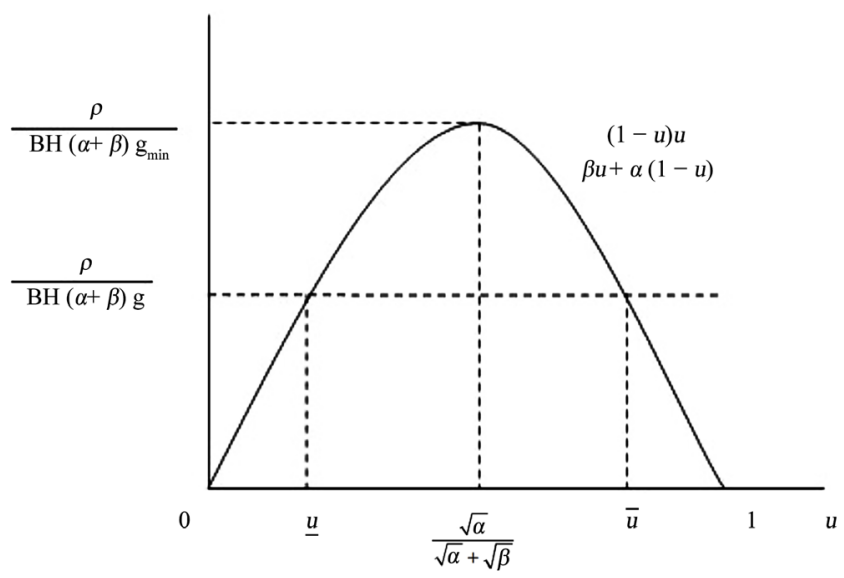

Figure 1. The optimal spending ratio on the research sector.

$$
\left(\frac{C}{Y}\right)^{*}=\frac{\frac{\alpha+\beta}{1-\alpha-\beta} H+\frac{\rho}{B u g}}{\frac{1-\beta}{\alpha} \frac{\alpha+\beta}{1-\alpha-\beta} \frac{H}{1-g}}
$$

We know that choosing $\bar{u}$ will cause consumer to have a lower consumption propensity.

We summarize the analysis above in the proposition below:

PROPOSITION 1. In the model of endogenous technological change with government spending, there exists a minimum overall government spending proportion to ensure the economy having an endogenous growth solution. Given a constant government spending ratio strictly larger than this minimum ratio, the government can choose either a higher spending ratio on research or a lower one. A government with the goal of high growth rate on the balanced growth path will prefer to spend more on the research sector. Then for a consumer, consumption propensity to income will be low, and for the government, the scale of the government spending tends to be larger.

\subsection{Analysis of the Dynamic Transition}

\subsubsection{Description of the Dynamic Transition Process}

We have showed that given the government spending ratio $g$, the spending proportion on the research sector and labor supply on the research sector will be chosen according to Equations (7) and (8). Then from

$$
\begin{aligned}
\left(\frac{Y}{K}\right)^{*} & =\frac{1-\beta}{1-\alpha-\beta} \frac{\alpha+\beta}{\alpha} \frac{B u g H}{1-g} \\
& =\eta^{\frac{\alpha+\beta-1}{1-\beta}} A^{\frac{\alpha+\beta}{1-\beta}}\left(H-H_{A}\right)^{\frac{\alpha}{1-\beta}}[(1-u) g]^{\frac{\beta}{1-\beta}} K^{\frac{1-\alpha-\beta}{1-\beta}}
\end{aligned}
$$

We have

$$
\left(\frac{A^{\alpha+\beta}}{K^{\alpha}}\right)^{*}=\frac{\left(\frac{1-\beta}{1-\alpha-\beta} \frac{\alpha+\beta}{\alpha} B u g H\right)^{1-\beta}}{(1-g)^{1-\beta} \eta^{\alpha+\beta-1}\left(H-H_{A}\right)^{\alpha}[(1-u) g]^{\beta}}
$$


But these two state variables $A$ and $K$ at the beginning do not need to satisfy

$$
\frac{A(0)^{\alpha+\beta}}{K(0)^{\alpha}}=\left(\frac{A^{\alpha+\beta}}{K^{\alpha}}\right)^{*}
$$

So there must be a dynamic transition process from the initial state to the balanced growing state.

Define

$$
q \equiv \frac{Y}{K}, p \equiv \frac{C}{K}
$$

the dynamic transition system for $p, q, u$ and $H_{A}$ can be derived from Equations (1) to (6).But we can simplify it by assuming that the government pursues not only the economic welfares but also the policy consistency. So the government prefers a stable choice of $u$ to varying it according to this system over time. Hence at the initial, the government will set $u=u^{*}$ for consistency, and then $H_{A}=H_{A}^{*}$ by Equations (7) and (8). This four-dimension system thus becomes

$$
\left\{\begin{array}{l}
\dot{q}=q\left[\frac{\alpha+\beta}{1-\beta} B u^{*} g H_{A}^{*}-\frac{\alpha}{1-\beta}(1-g) q+\frac{\alpha}{1-\beta} p\right] \\
\dot{p}=p\left[-\rho-\frac{\alpha}{1-\beta}(1-g) q+p\right]
\end{array}\right.
$$

And this system describes the dynamic transition process after the government controlling $u$.

From $\dot{q}=0, \dot{p}=0$, the steady state is

$$
\begin{gathered}
q^{*}=\frac{1-\beta}{1-\alpha-\beta} \frac{\alpha+\beta}{\alpha} \frac{B u^{*} g H}{1-g} \\
p^{*}=\rho+\frac{\alpha+\beta}{1-\alpha-\beta} B u^{*} g H
\end{gathered}
$$

To analyze the stability of this system, we linearize it around the steady state:

$$
\left(\begin{array}{c}
\dot{q} \\
\dot{p}
\end{array}\right)=\left(\begin{array}{cc}
-\frac{\alpha}{1-\beta}(1-g) q^{*} & \frac{\alpha}{1-\beta} q^{*} \\
-\frac{\alpha}{1-\beta}(1-g) p^{*} & p^{*}
\end{array}\right)\left(\begin{array}{c}
q-q^{*} \\
p-p^{*}
\end{array}\right)
$$

Let $\lambda_{1}, \lambda_{2}$ be the eigenvalues of the system, then

$$
\begin{gathered}
\lambda_{1}+\lambda_{2}=p>0 \\
\lambda_{1} * \lambda_{2}=-\frac{(1-\alpha-\beta)^{\alpha}}{(1-\beta)^{2}}(1-g) p^{*} q^{*}<0
\end{gathered}
$$

Hence one of the eigenvalues is negative (assuming $\lambda_{1}$ ), the other is positive (assuming $\lambda_{2}$ ), and the system is stable.

\subsubsection{Short-Run Analysis}

The dynamic transition system above gives us another perspective to analyze the government's choice between $\underline{u}$ and $\bar{u}$ : the government may weight more on 
the short-run welfare than on the long-run growth rate. So in this subsection we are interested in the difference of $p(0)$ between $u=\underline{u}$ and $u=\bar{u}$.

Base on the linearized system (10), the purely stable adjustment path of $p(0)$, starting from the initial state

$$
q(0)=\eta^{\frac{\alpha+\beta-1}{1-\beta}} \alpha^{\frac{\alpha}{1-\beta}} g^{\frac{\beta}{1-\beta}} H^{\frac{\alpha}{1-\beta}} \frac{(1-u)^{\frac{\alpha+\beta}{1-\beta}}}{[\alpha(1-u)+\beta u]^{\frac{\alpha}{1-\beta}}}\left[\frac{A(0)^{\alpha+\beta}}{K(0)^{\alpha}}\right]^{\frac{1}{1-\beta}}
$$

is described by

$$
p(0)=p^{*}+\Lambda\left[q(0)-q^{*}\right]
$$

where

$$
\Lambda=\frac{\alpha(1-g) p^{*}}{(1-\beta)\left(p^{*}-\lambda_{1}\right)}<0
$$

Given a constant $g$, we assume that the matrix from system (10) does not change between $u=\bar{u}$ and $u=\underline{u}$, and thus the eigenvalues are still $\lambda_{1}, \lambda_{2}$. It follows immediately that $\Lambda$ is constant. Then since

$$
\begin{aligned}
& \left.p(0)\right|_{u=\bar{u}}-\left.p(0)\right|_{u=\underline{u}} \\
& =\left(1-\Lambda \frac{1-\beta}{\alpha} \frac{1}{1-g}\right) \frac{\alpha+\beta}{1-\alpha-\beta} \operatorname{Bg} H(\bar{u}-\underline{u})+\Lambda\left[\left.q(0)\right|_{u=\bar{u}}-\left.q(0)\right|_{u=\underline{u}}\right]
\end{aligned}
$$

and the first term of the right hand are positive, two possibilities can be obtained as follows:

1) $\alpha \leq \beta$

Then

$$
\left.q(0)\right|_{u=\bar{u}} \leq\left. q(0)\right|_{u=\underline{u}}
$$

So

$$
\left.p(0)\right|_{u=\bar{u}}>\left.p(0)\right|_{u=\underline{u}}
$$

The government always prefers $u=\bar{u}$, that is, the government will choose the high spending ratio on the research sector.

2) $\alpha>\beta$

Then

$$
\left.q(0)\right|_{u=\bar{u}}>\left.q(0)\right|_{u=\underline{u}}
$$

If

$$
\begin{aligned}
\frac{A(0)^{\alpha+\beta}}{K(0)^{\alpha}}>- & \frac{(1-\beta)(\sqrt{\alpha}+\sqrt{\beta})^{\beta-\alpha}(\sqrt{\alpha})^{\alpha} \rho}{(\alpha+\beta) \alpha^{\frac{\alpha+1-\beta}{1-\beta}}(\sqrt{\beta})^{\beta} \eta^{\frac{\alpha+\beta-1}{1-\beta}} H^{\frac{\alpha}{1-\beta}} \frac{1}{1-g}} \\
& + \text { terms_that_are_constant }
\end{aligned}
$$

Then

$$
\left.p(0)\right|_{u=\bar{u}}<\left.p(0)\right|_{u=\underline{u}}
$$


the government prefers $u=\underline{u}$. Otherwise it will still choose the high spending ratio on the research sector.

On one hand, the condition above in countries with high overall government spending ratio is easily satisfied, so these countries tend to choose the low spending ratio on the research sector to consume more instantaneously. On the other hand, the condition above helps us understand why some governments, especially in developing countries like China, prefer the low spending ratio on the research sector. Since the stock of knowledge has a positive externality, and the designs are nontrivial that can be costless replicated, developing countries at the beginning have endowed a relatively high stock of knowledge and a relatively low stock of physical capital, and thus have a larger ratio of $\frac{A(0)^{\alpha+\beta}}{K(0)^{\alpha}}$. So marginal return from physical capital accumulation in the final output sector exceeds that from knowledge design in the research sector, the government will weigh more on the production than on the innovation. We summarize the analysis above in the proposition below:

PROPOSITION 2. There would be a dynamic transition process from the initial state to the equilibrium. The government is then likely to take the short-run welfare into account. When the endowed stock of knowledge is substantive and the physical capital just starts to be accumulated, the government will choose the low spending ratio on the research sector.

\section{Conclusions}

The paper incorporates government expenditure into Romer's model of endogenous technological change in a manner that government expenditure not only is an input to private production but also helps increase the productivity in the research sector. First, this model derives a minimum overall government spending proportion to sustain an endogenous growth, and this minimum proportion depends on the patience and the labor supply of the economy.

Then it follows that the government can choose between two spending ratios on the research sector to maintain endogenous growth, and thus influences the economy differently. Both long-run and short-run analysis can be taken into account to decide which one to choose. On the balanced growth path, the higher spending ratio on the research sector brings higher growth rate, and is preferred. But since there is a dynamic transition process for the economy from initial state to the balanced growth path, the lower one will be optimal for a country with relatively high endowed stock of knowledge and relatively low endowed physical capital.

The analysis above only considers a case of central planner who can distribute all the resources appropriately into different sectors, so the model can be extended in the future to consider the optimal allocation of spending in a decentralized economy, and certain compilations between these two economies can be made. 
Based on the conclusion of the paper, some empirical studies should be carried on to find whether some countries spend the optimal ratio on the research sector. The model also provides a criterion for future analysis to compare between different countries the ratio of stock of knowledge and physical capital.

\section{References}

[1] Barro, R.J. (1990) Government Spending in a Simple Model of Endogenous Growth. Journal of Political Economy, 98, S103-S125.

[2] Romer, P.M. (1990) Endogenous Technological Change. Journal of Political Economy, 98, S71-S102. https://doi.org/10.1086/261725 